Evol ut i on from a ferromanet i c to a spi n-gl ass regi re in the spi nel-type $\mathrm{Cu}(\mathrm{Cr} 1-\mathrm{xTi} x) 2 \mathrm{~S} 4$

\begin{tabular}{|l|l|}
\hline 著者 & $\begin{array}{l}\text { KARI YA Fumi hi ro, EBI SU Shuj i , NAGATA } \\
\text { Shoi chi }\end{array}$ \\
\hline $\begin{array}{l}\text { j our nal or } \\
\text { publ i cat i on ti tl e }\end{array}$ & Jour nal of sol i d st at e chemi st ry \\
\hline vol une & 182 \\
\hline nunber & 3 \\
\hline page r ange & $608-616$ \\
\hline year & $2009-03$ \\
\hline URL & ht t p: //hdl . handl e. net /10258/487 \\
\hline
\end{tabular}


Evol ut i on from a ferromanet i c to a spi n-gl ass regi re in the spi nel-type $\mathrm{Cu}(\mathrm{Cr} 1-\mathrm{xTi} x) 2 \mathrm{~S} 4$

\begin{tabular}{|l|l|}
\hline 著者 & $\begin{array}{l}\text { KARI YA Fumi hi ro, EBI SU Shuj i , NAGATA } \\
\text { Shoi chi }\end{array}$ \\
\hline $\begin{array}{l}\text { j our nal or } \\
\text { publ i cat i on ti tl e }\end{array}$ & Jour nal of sol i d st at e chemi st ry \\
\hline vol une & 182 \\
\hline nunber & 3 \\
\hline page r ange & $608-616$ \\
\hline year & $2009-03$ \\
\hline URL & ht t p: //hdl . handl e. net /10258/487 \\
\hline
\end{tabular}




\title{
Evolution from a ferromagnetic to a spin-glass regime in the spinel-type $\mathrm{Cu}\left(\mathrm{Cr}_{1-x} \mathrm{Ti}_{x}\right)_{2} \mathrm{~S}_{4}$
}

\author{
Fumihiro Kariya, Shuji Ebisu, \\ Shoichi Nagata \\ Department of Materials Science and Engineering, Muroran Institute of Technology, 27-1 \\ Mizumoto-cho, Muroran, Hokkaido, 050-8585 Japan
}

\begin{abstract}
Successive changes from ferromagnetic, re-entrant mixed, to spin-glass regime have been manifestly found with increasing Ti-composition $x$ in the quaternary spinel-type $\mathrm{Cu}\left(\mathrm{Cr}_{1-x} \mathrm{Ti}_{x}\right)_{2} \mathrm{~S}_{4}$ system. The Curie temperature $T_{\mathrm{c}}$ decreases steeply with increasing $x$ and this transition becomes ill-defined around $x=0.47$. Two distinct transitions appear below $T_{\mathrm{c}}$ over the range $x$ $=0.40-0.47$. Coexistence of the ferromagnetism and spin-glass order would be observed below the Gabay and Toulouse transition $\left(T_{\mathrm{GT}}\right)$, owing to freezing of the transverse-spin components without changing of the ferromagnetic order parameter. Finally, at a yet lower temperature de Almeida-Thouless transition $\left(T_{\mathrm{AT}}\right)$, the longitudinal-spin component freezes randomly at which an irreversibility arises between zero-field-cooled (ZFC) and field-cooled (FC) magnetizations. Over the range of $0.47 \leq x \leq 0.85$, a cusp of the $\mathrm{ZFC}$ magnetization is seen at $T_{\mathrm{g}}$ like conventional spin-glass. Specimens with $x \geq 0.90$ remain paramagnetic down to $2.0 \mathrm{~K}$. A magnetic phase diagram between $T$ versus $x$ has been obtained experimentally. The values of the multi critical point in 100 Oe is detected to be $x=0.47$ and $T=7.40 \mathrm{~K}$. The low field magnetization and the phase diagram are satisfactorily explained by the theory of Gabay and Toulouse on the basis of Heisenberg isotropic vector spin model rather than the Ising spin model.
\end{abstract}

Key words: Spinel-type $\mathrm{Cu}\left(\mathrm{Cr}_{1-x} \mathrm{Ti}_{x}\right)_{2} \mathrm{~S}_{4}$, Spin-glass, Magnetic phase diagram

Email addresses: naga-sho@mm .muroran-it.ac.jp (Shoichi Nagata) 


\section{Introduction}

A spinel $\mathrm{CuCr}_{2} \mathrm{~S}_{4}$ is a metallic ferromagnet with a Curie temperature $T_{\mathrm{c}}$ $\simeq 380 \mathrm{~K} .(1 ; 2 ; 3 ; 4 ; 5 ; 6 ; 7 ; 8 ; 9 ; 10 ; 11 ; 12 ; 13 ; 14 ; 15 ; 16 ; 17 ; 18 ; 19 ; 20$ : $21 ; 22 ; 23 ; 24 ; 25 ; 26)$ The formula unit has a net magnetic moment close to $5.0 \mu_{\mathrm{B}}$. The $\mathrm{CuCr}_{2} \mathrm{~S}_{4}$ has the mixed valence as $\mathrm{Cu}^{+} \mathrm{Cr}^{3+} \mathrm{Cr}^{4+} \mathrm{S}_{4}{ }^{2-}$, here $\mathrm{Cr}^{3+}$ ion with $3 \mu_{\mathrm{B}}$ and $\mathrm{Cr}^{4+}$ ion with $2 \mu_{\mathrm{B}}$, and a $\mathrm{Cu}^{+}$ion has the closed shell, where all the $\mathrm{Cr}$ ions align parallel each other, as a results the formula unit of $\mathrm{CuCr}_{2} \mathrm{~S}_{4}$ has a net magnetic moment of $5 \mu_{\mathrm{B}}$. The metallic conduction and the ferromagnetism have been attributed to double exchange between $\mathrm{Cr}^{3+}$ and $\mathrm{Cr}^{4+}$ ions. $(2 ; 3 ; 10 ; 19 ; 22)$

On the other hand, $\mathrm{CuTi}_{2} \mathrm{~S}_{4}$ has also the spinel structure and shows metallic properties without any magnetic anomaly. $(27 ; 28 ; 29 ; 30 ; 31 ; 32)$ Considerable studies of the mixed spinel $\mathrm{Cu}\left(\mathrm{Cr}_{1-x} \mathrm{Ti}_{x}\right)_{2} \mathrm{~S}_{4}$ have been also made by the many researchers. $(33 ; 34 ; 35 ; 36 ; 37 ; 38 ; 39)$ These previous results of $\mathrm{Cu}\left(\mathrm{Cr}_{1-x} \mathrm{Ti}_{x}\right)_{2} \mathrm{~S}_{4}$, however, have less rich data to understand the physics of magnetism. A systematic and the dedicated experimental work has been now carried out for the quaternary $\mathrm{Cu}\left(\mathrm{Cr}_{1-x} \mathrm{Ti}_{x}\right)_{2} \mathrm{~S}_{4}$ in order to understand the magnetic state.

High-purity specimens of $\mathrm{Cu}\left(\mathrm{Cr}_{1-x} \mathrm{Ti}_{x}\right)_{2} \mathrm{~S}_{4}$ have been successfully prepared. The atoms of $\mathrm{Cr}$ and $\mathrm{Ti}$ are distributed randomly in the octahedral $B$-site. (40) Present study is motivated by the preceding works for the mixed spinel $\mathrm{CuCrZrS}_{4}$. $(41 ; 42 ; 43)$ The common feature of this families of the mixed spinel compounds $\mathrm{Cu}\left(\mathrm{Cr}_{1-x} M_{x}\right)_{2} \mathrm{~S}_{4}$ (where $M=\mathrm{Ti}, \mathrm{Zr}$, Hf) is that the substitution of $M$ element for Cr atom in the $\mathrm{B}$-sites yields richness of the re-entrant spin-glass phase.

Temperature dependence of the low-field magnetization has been studied systematically down to $2.0 \mathrm{~K}$ in the present work. The increase of Ticomposition $x$ leads to a successive change of the magnetic state, from a dominant ferromagnetic, a re-entrant mixed, a typical spin-glass, and finally to a pure paramagnetic state. The random distribution of $\mathrm{Ti}$ atom in $B$-sites can cause magnetic competing interactions.

The present experimental results can be explained reasonably by the theory of Gabay and Toulouse (G-T) (44) on the basis of Heisenberg model, which is different from the conclusions from the Ising model. (45; 46) The 
Heisenberg model calculation on the basis of this $G-T$ theory predicts that there should be two distinct changes of behavior below $T_{c}$. (44) The system passes from paramagnetic to ferromagnetic at $T_{\mathrm{c}}$, then at a lower temperature G-T transition ( $T_{\mathrm{GT}}$ ), the transverse-moment components of each spin begin to freeze and coexist with the ferromagnetic state without changing of the ferromagnetic order parameter, and at a yet lower temperature de Almeida-Thouless $(\mathrm{A}-\mathrm{T})$ transition $\left(T_{\mathrm{AT}}\right)(47)$, the longitudinal-spin component freezes randomly $\left(T_{\mathrm{AT}}<T_{\mathrm{GT}}<T_{\mathrm{c}}\right)$. At $T_{\mathrm{AT}}$, a crossover from weakly irreversible to strongly irreversible behavior takes place, where an irreversible effect between zero-field-cooled (ZFC) and field-cooled (FC) magnetization appears. (48)

The study of spin-glass has been of great theoretical and experimental interest since last 36 years. $(49 ; 50 ; 51 ; 52 ; 53)$ The magnetic phase diagram, between magnetic state and the concentration of magnetic element, however, has been less extensively studied for the spin-glass system. Several magnetic phase diagrams of spin-glass system have been available. $(54 ; 55 ; 56 ; 57 ; 58)$ To the best our knowledge the magnetic phase diagram concerned with the G-T transition has not been reported so far, the experimental evidence for the G-T transition is not given either, only an exception is the observation of hyperfine field anomaly in the Mössbauer measurement for $A u F e$ specimen. (59) Any other experiments involved with the magnetic phase diagram have not yet been applied directly to the G-T theory of the spin-glass, so we can point out the possibility of a connection.

The interplay between theoretical and experimental studies depends on reliable specimens. An essential advantage of this system is to provide a restricted stage for the performance on the spin frustration. The precise control of the exact Cr:Ti ratio $x$ can be made on the $B$-site sublattice of the spinel structure. Consequently, the fairly accurate results of composition dependence of the competing interactions is promising and guaranteed. On the other hand, it is rather hard to eliminate the inhomogeneity for the system of metallic alloy specimens in the some past workers.

The aim of this paper is to present a magnetic phase diagram between $T$ versus $\mathrm{Ti}$ composition $x$ for $\mathrm{Cu}\left(\mathrm{Cr}_{1-x} \mathrm{Ti}_{x}\right)_{2} \mathrm{~S}_{4}$. We will discuss new results of the coexistence of the ferromagnetic and spin-glass state. The substituted quaternary spinel system $\mathrm{Cu}\left(\mathrm{Cr}_{1-x} \mathrm{Ti}_{x}\right)_{2} \mathrm{~S}_{4}$ is able to exemplify a systematic investigation of the $\mathrm{G}-\mathrm{T}$ and the $\mathrm{A}-\mathrm{T}$ transitions. The present study is mainly concerned with the experimental finding from the dc magnetization measurements, while the dynamical magnetic property, thermal and electri- 
cal properties are not given. The detailed microscopic magnetic interactions will not be discussed in this paper because of lack of information from the microscopic results.

\section{Experimental methods}

The polycrystalline specimens were prepared by a solid-state reaction. Mixtures of high-purity fine powders of $\mathrm{Cu}$ (purity $99.99 \%$, melting point $1356 \mathrm{~K}), \mathrm{Cr}(99.99 \%, 2148 \mathrm{~K}), \mathrm{Ti}(99.9 \%, 1941 \mathrm{~K})$, and S $(99.999 \%, 392 \mathrm{~K})$ with nominal stoichiometry were heated in sealed quartz ampoules to 1223 $\mathrm{K}$ and kept at this temperature for 5 days. Samples were annealed at 673 $\mathrm{K}$ for $4 \mathrm{~h}$. The prepared specimens with grayish black in color were found to be stable under air after the synthesis. The identification of the crystal structure and the determination of the lattice constants were carried out by $\mathrm{X}$-ray powder diffraction using $\mathrm{Cu} \mathrm{K} \alpha$ radiation at room temperature.

The dc magnetization measurements of powder specimens were performed with a quantum design superconducting quantum interference device (rfSQUID) magnetometer over the temperature range 2.0 - $350 \mathrm{~K}$. For many specimens, the experimental results showed the appearance the irreversible effect between the ZFC (initial susceptibility, after cooling the sample to the lowest temperature in zero field, a field was applied and the data were taken with increasing temperature) and the $\mathrm{FC}$ magnetizations.(48) The demagnetizing field corrections have not been applied to any of our magnetic data.

\section{Results and discussion}

\subsection{Lattice contant}

$\mathrm{X}$-ray powder diffraction patterns at room temperature give evidence that $\mathrm{Cu}\left(\mathrm{Cr}_{1-x} \mathrm{Ti}_{x}\right)_{2} \mathrm{~S}_{4}$ has the spinel type structure. The X-ray data have been analyzed assuming a cubic spinel structure with the space group $F d \overline{3} m$ (No. 227). Samples with $x<0.15$ were not pure phase. The study of the lower value of $x$ than 0.15 has a disadvantage that the preparation of single phase samples is more difficult. Representative diffraction profiles are shown in Fig. 1. The $\mathrm{X}$-ray diffraction study verifies that the reliability factor gives minimum only for a random distribution of $\mathrm{Cr}$ and $\mathrm{Ti}$ atoms in the $B$-site.(40) The lattice constant, $a$, obtained by the least square method, varies as shown in Fig. 2 at room temperature. The lattice constant increases linearly with 


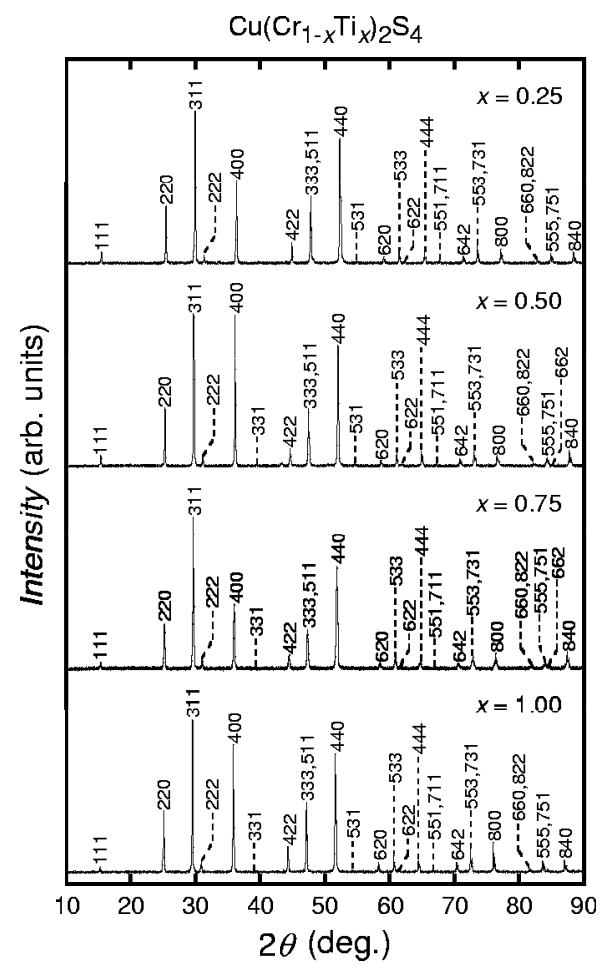

Figure 1: X-ray powder diffraction profiles for $x=0.25,0.50,0.75$, and 1.00 at room temperature. 


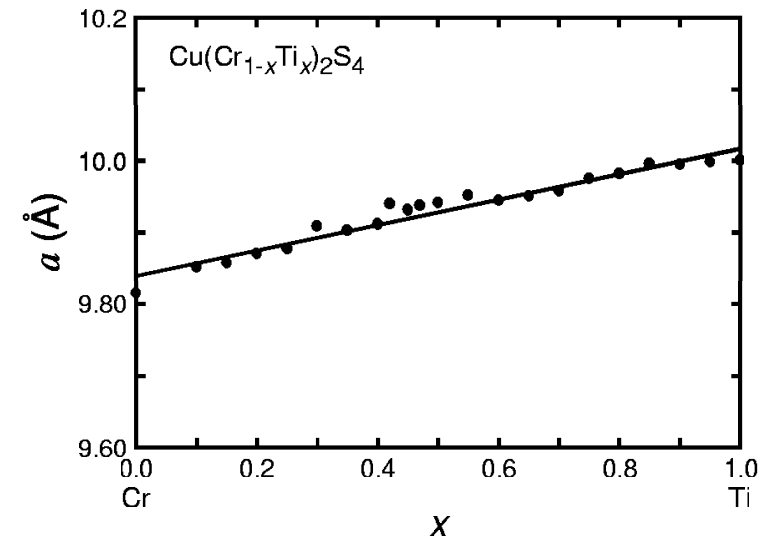

Figure 2: The lattice constant $a$ as a function of Ti composition $x$ at room temperature. 
increasing Ti substitution $x$ and obeys the Vegard's law. The unit cell size is reported to be $a=9.814 \AA$ for $\mathrm{CuCr}_{2} \mathrm{~S}_{4},(4) a=10.000 \AA$ for $\mathrm{CuTi}_{2} \mathrm{~S}_{4},(31)$ respectively.

\subsection{Temperature dependences of magnetization in a field of $100 \mathrm{O}_{\epsilon}$}

Figures from 3 to 6 display the temperature dependences of the magnetization in a constant field of 100 Oe down to $2.0 \mathrm{~K}$ for $\mathrm{Cu}\left(\mathrm{Cr}_{1-x} \mathrm{Ti}_{x}\right)_{2} \mathrm{~S}_{4}$. The magnetization as a function of temperature demonstrates the spin-glass characteristics with changing the value of $x$. The experimental results do not yield a simple diluted ferromagnetism with increasing Ti substitution, but the variation of the magnetic state is fairly complex and reflects the subtlety of magnetic interaction. On beginning from a predominant ferromagnetic state, a re-entrant mixed, a typical spin-glass, and to a paramagnetic state, the sequence of events seems to be fairly natural. The evolutional change of the magnetic state has been found in Figs. $3-6$, as discussed below.

\subsection{The Curie-Weiss law}

The high temperature susceptibility can be fitted to a modified CurieWeiss law; $\chi=C /(T-\theta)+\chi_{0}$, where $\chi_{0}$ is the temperature independent term, $C$ the Curie constant, and $\theta$ the Weiss temperature. Figure 7 shows the temperature dependences of the inverse susceptibility $\chi^{-1}=(M / H)^{-1}$ for $x=0.50$ and 0.55 , where the low values of $\theta$ are 1.12 and $4.38 \mathrm{~K}$, respectively. Figure 8 presents the inverse susceptibilities for $0.45 \leq x \leq 0.75$. The summary of the magnetic properties from the Curie-Weiss law is listed in Table 1. The value of effective magnetic moment $p_{\text {eff }} \mathrm{Cr}$-atom ${ }^{-1}$ is extracted, assuming that only $\mathrm{Cr}$ atoms possess a localized magnetic moment, while $\mathrm{Cu}$, Ti and $\mathrm{S}$ atoms have no magnetic moment. As can be seen in Table 1, this evaluation can lead a conclusion that $\mathrm{Cr}^{3+}$ ion octahedrally surrounded by $\mathrm{S}$ ions yields a half-filled $t_{2 q}$ triplet with spin only value of $S=3 / 2$. The asymptotic Weiss temperature $\theta$ has small value for $x=0.47,0.50$, and 0.55 , reflecting the nature of competing interactions between ferro- and antiferromagnetic exchange interactions in the typical spin-glass. Figure 9 displays the composition dependence of the magnitude of $\theta$. The predominant ferromagnetic state is attained for $0.00 \leq x \leq 0.35$. The composition dependence of $\theta$ shows a round peak around $x=0.75$, nevertheless the value of $p_{\text {eff }} \mathrm{Cr}-$ atom $^{-1}$ is approximately constant and close to the expected spin only value of $3.87 \mu_{\mathrm{B}}$ per $\mathrm{Cr}^{3+}$ over all the composition range. It should be noted that 


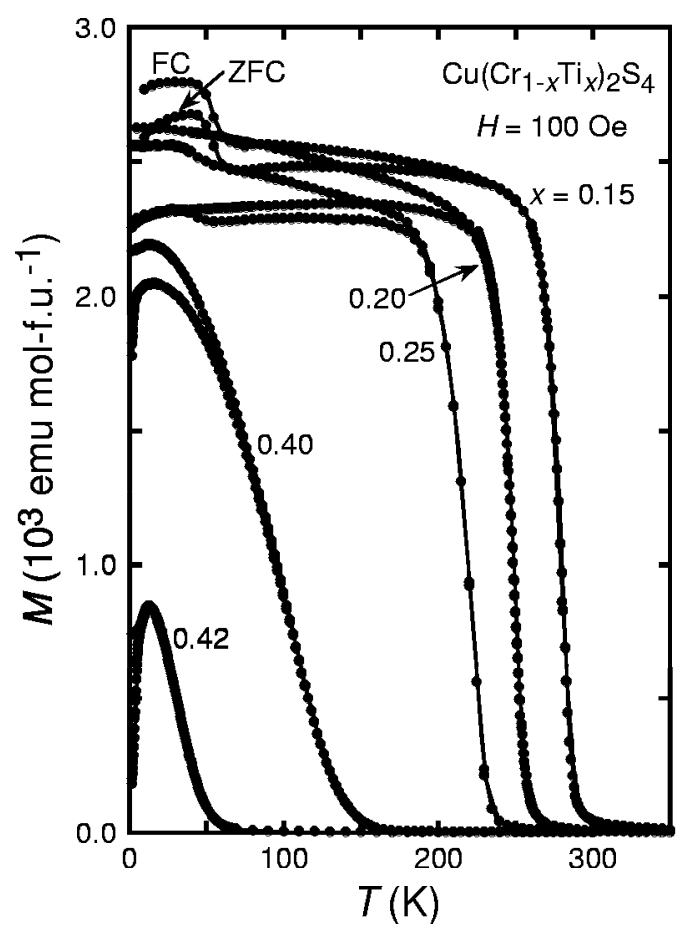

Figure 3: Temperature dependences of magnetization for $\mathrm{Cu}\left(\mathrm{Cr}_{1-x} \mathrm{Ti}_{x}\right)_{2} \mathrm{~S}_{4}$ in a constant magnetic field of 100 Oe for $0.15 \leq x \leq 0.42$. The difference between field-cooled (FC) and zero-field cooled (ZFC) magnetizations are indicated. It is important to notice that the magnetization is not saturated at 100 Oe, but is under the influence of displacement of multidomain in the predominant ferromagnetic state, in particular for $0.15<x<0.25$. 


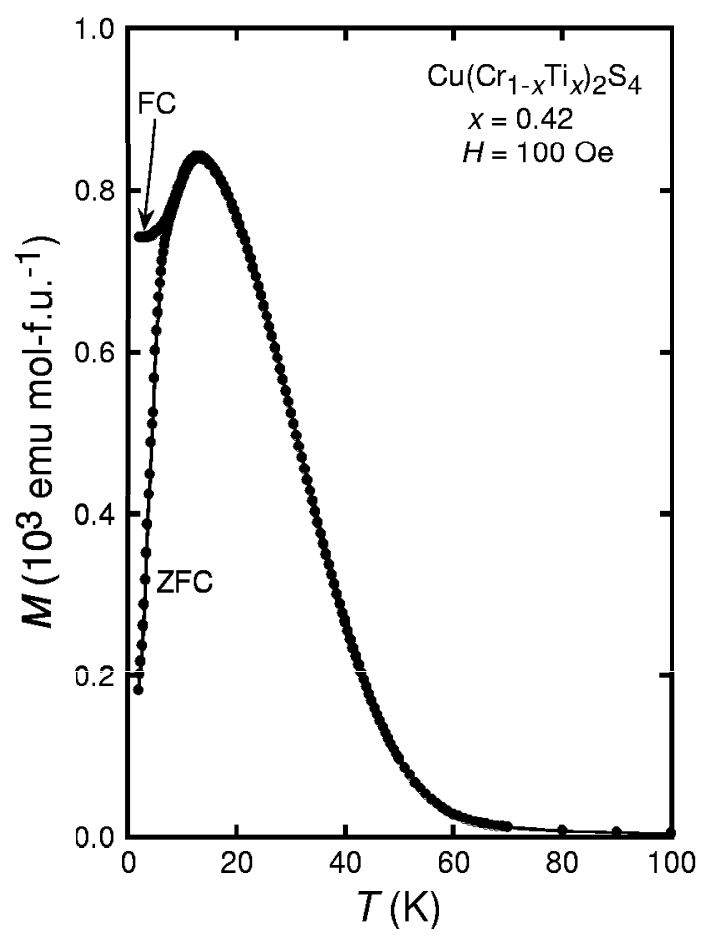

Figure 4: Magnetization as a function of temperature for $x=0.42$ in a constant field of 100 Oe. The ferromagnetic transition is estimated to be $55 \mathrm{~K}$ as listed in Table 2. Below $T_{\mathrm{GT}}=13.0 \mathrm{~K}$ of the peak temperature, the coexistence of the ferromagnetic and spin-glass phases would be realized. At a yet lower temperature $T_{\mathrm{AT}}=6.6 \mathrm{~K}$, an irreversible effect between a zero-field-cooled (ZFC) and a field-cooled (FC) magnetization appears. Two different regions of SGI and SGII are detected, see text. 


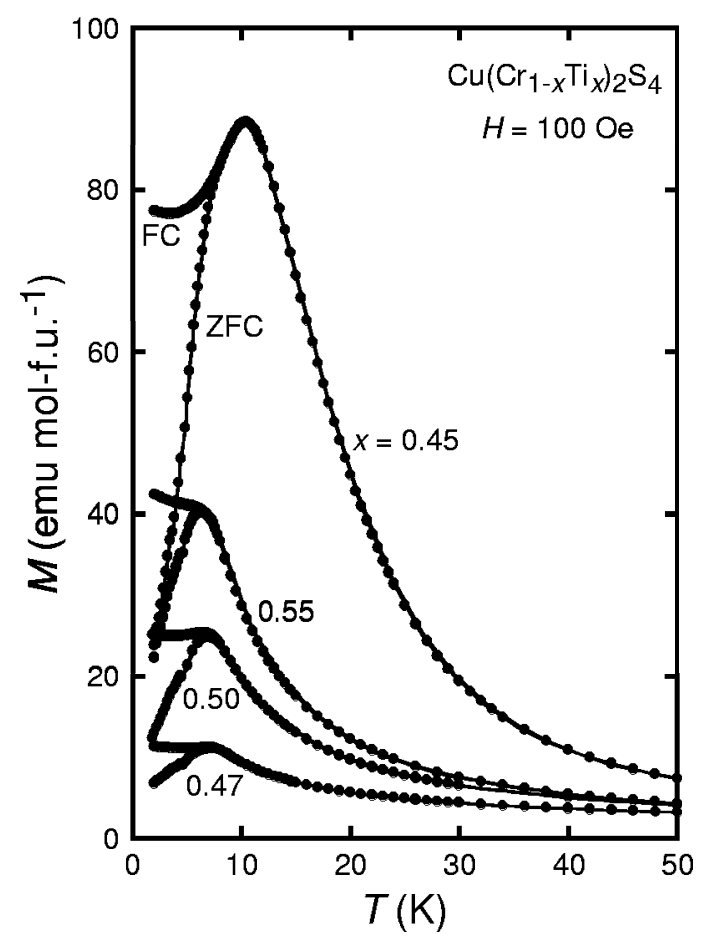

Figure 5: Temperature dependences of magnetization for $\mathrm{Cu}\left(\mathrm{Cr}_{1-x} \mathrm{Ti}_{x}\right)_{2} \mathrm{~S}_{4}$ in a constant field of 100 Oe for $0.45 \leq x \leq 0.55$. 


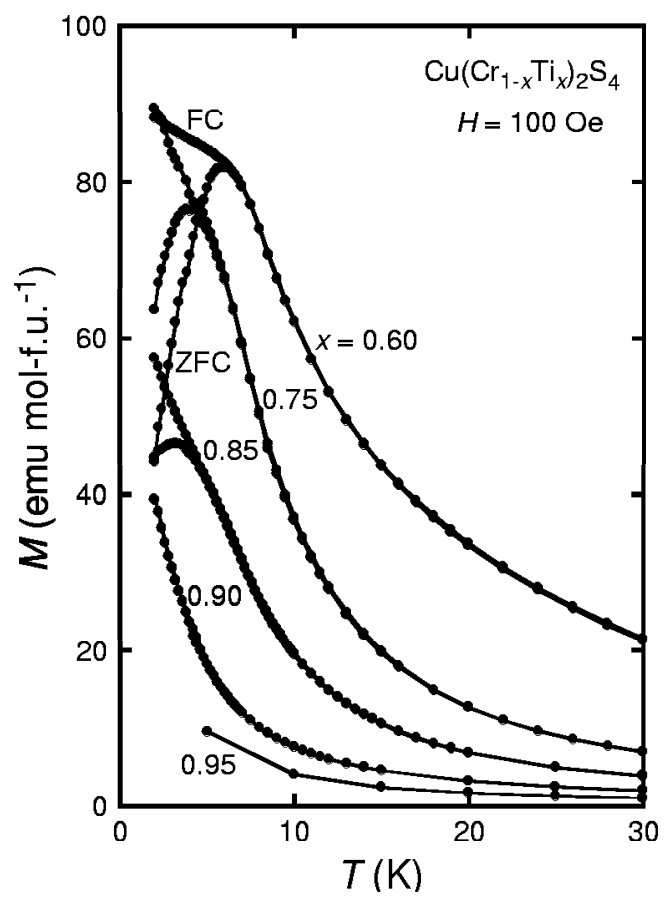

Figure 6: Temperature dependences of magnetization for $\mathrm{Cu}\left(\mathrm{Cr}_{1-x} \mathrm{Ti}_{x}\right)_{2} \mathrm{~S}_{4}$ in a constant field of 100 Oe for $0.60 \leq x \leq 0.95$. 


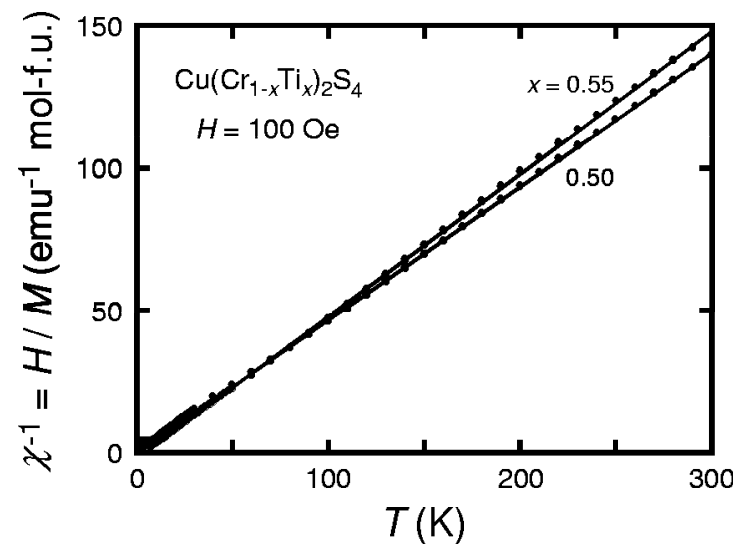

Figure 7: Inverse magnetic susceptibilities $\chi^{-1}=(M / H)^{-1}$ for the specimens with $x=$ 0.50 and 0.55 . 


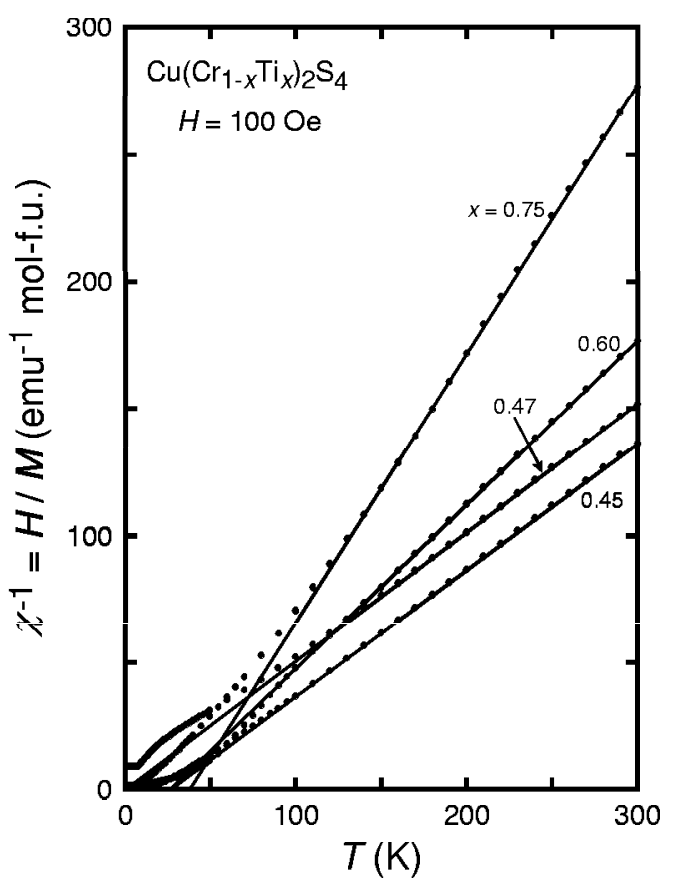

Figure 8: Temperature dependences of the inverse magnetic susceptibility $\chi^{-1}=(M / H)^{-1}$ for $0.45 \leq x \leq 0.75$ in a constant field of 100 Oe. 


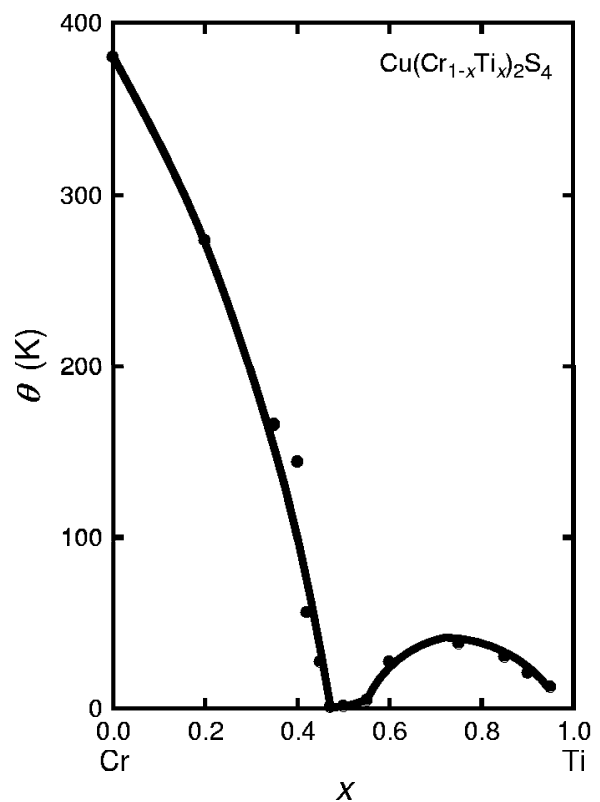

Figure 9: Composition dependence of the asymptotic Weiss temperature $\theta$. 
the results of Fig. 9 does not vary in the monotonic way with $x$. As indicated in Fig. 8, $\chi^{-1}$ cannot be fit to a single straight line over the wide temperature rage for $0.45 \leq x \leq 0.75$, then we have taken the value of $\theta$ from the higher temperature region as the asymptotic Weiss temperature. Therefore, the value of $\theta$ for $0.45 \leq x \leq 0.75$ in Fig. 9 has still uncertainty. The discussion along with this behavior is involved in the later section.

\subsection{Variation of the ferromagnetism}

Figure 3 shows the temperature dependences of the magnetization in a constant field of 100 Oe for $0.15 \leq x \leq 0.42$. The magnetization increases abruptly, showing the ferromagnetic order with decreasing temperature. The specimens with $x \leq 0.35$ exhibit predominant ferromagnetism. The result of $x=0.35$ is not shown in Fig. 3 for avoiding overlap of the data in the lower temperature region. The difference between the $\mathrm{ZFC}$ and the $\mathrm{FC}$ magnetizations is seen, which is mainly the traces of the influence of domain-wall blocking in the multidomain structure. The low magnetic field of 100 Oe cannot make saturate the magnetization. Irreversible domain boundary displacement is caused through the different magnetic treatments of the $\mathrm{ZFC}$ and the $\mathrm{FC}$ for the specimens.

A broad hump of the magnetization arises from $x=0.15$ and 0.25 below around $50 \mathrm{~K}$. The origin of this hump is still unclear at present, whereas this unusual hump might be inferred from an inherent property of these specimens related to frustration effect on the domain-wall blocking. It is essentially different from another $\mathrm{Cr}$ spinel $\mathrm{Cu}_{1-x} \mathrm{Zn}_{x} \mathrm{Cr}_{2} \mathrm{Se}_{4},(60)$ despite an apparently similarity of the hump of this sample. The origin of the broad hump at low temperatures was attributed to a non-trivial magnetic structure of $\mathrm{Cr}$ moments such as the conical spin structure in $\mathrm{Cu}_{1-x} \mathrm{Zn}_{x} \mathrm{Cr}_{2} \mathrm{Se}_{4}$. This explanation is not justified and is likely to be ruled out for the present system, $\mathrm{Cu}\left(\mathrm{Cr}_{1-x} \mathrm{Ti}_{x}\right)_{2} \mathrm{~S}_{4}$, from judging that the occupation of $\mathrm{Cr}$ magnetic moment on the $B$-site is diluted by non magnetic $\mathrm{Ti}$ substitution. When the composition $x$ becomes higher, the hump would change apparently from the hump anomaly to the re-entrant anomaly. These humps indicate a sign and precursor of re-entrant spin-glass state, where random fields survive into lower temperatures and cause this anomaly.

Figure 10, as a representative result, indicates the temperature dependence of the magnetization and the inverse susceptibility for $x=0.40$ in a constant field of 100 Oe. Here, the susceptibility is defined as $\chi=M / H$. As seen in Fig. 10 the magnetization as a function of temperature is rather 


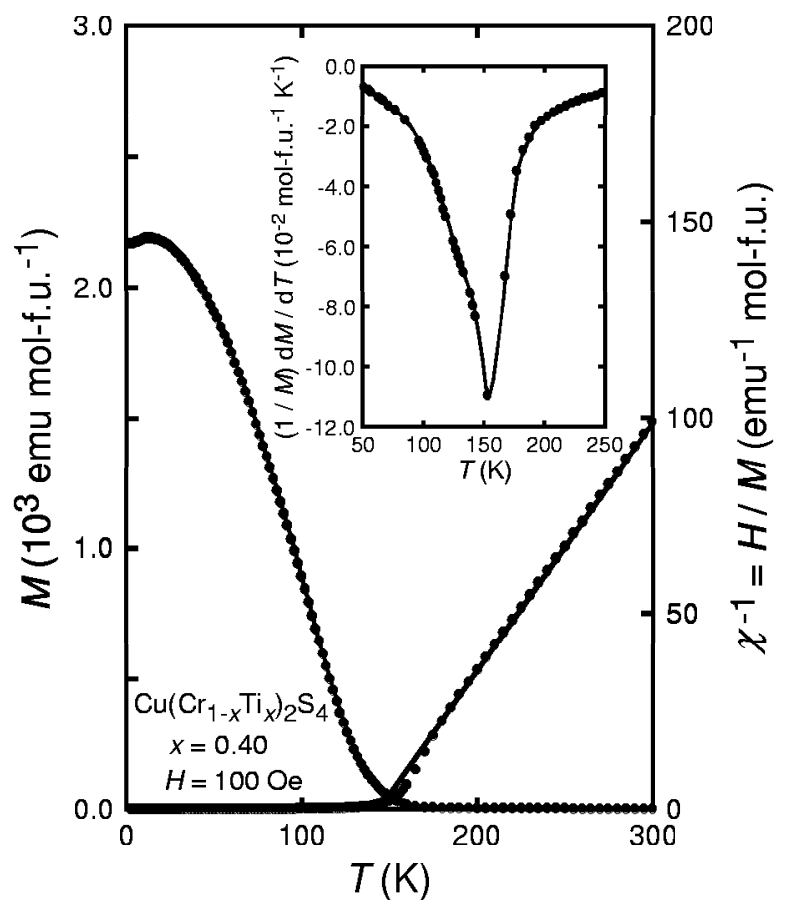

Figure 10: Magnetization and the inverse magnetic susceptibility $\chi^{-1}=(M / H)^{-1}$ as a function of temperature in a constant field of 100 Oe for $x=0.40$. The temperature derivative $(1 / M) d M / d T$ is presented in the inset. 
slender below $153 \mathrm{~K}$ due to the diluted magnetic concentration with $x=0.40$ than that of standard ferromagnets.

An analysis of derivative of $M$ with respect to $T$ was employed to determine the Curie point $T_{c}$. Here, the value of the derivative is normalized by the magnitude of the magnetization $M$. The inset in Fig. 10 shows a temperature derivative $(1 / M) d M / d T$ curve as a function of temperature for $x=0.40$. The temperature at the negative peak of $(1 / M) d M / d T$ curve is given to be approximately $153 \mathrm{~K}$. This inflection point can correspond to the Curie point of ferromagnetic order, above which the spontaneous magnetization disappears. The high temperature susceptibility can be fitted to the modified Curie-Weiss law. The asymptotic Curie temperature $\theta$ is 144 $\mathrm{K}$ and $p_{\text {eff }} \mathrm{Cr}$-atom ${ }^{-1}$ is $3.25 \mathrm{Cr}$-atom ${ }^{-1}$. The value of $\theta$ with $x=0.40$ is somewhat less than that of the inflection points of $(1 / M) d M / d T$. The values of composition dependence of the negative peak of $(1 / M) d M / d T$ are listed in Table 2.

Figure 11 demonstrates the temperature dependences of the $(1 / M) d M / d T$ for $x=0.42$ and 0.40 . This analysis allows us to determine an estimate of the ferromagnetic transition point $T_{\mathrm{c}}$. A characteristic feature of these results of Figs. $3-5$ is the re-entrant phenomena, that is, the high-temperature ferromagnetism giving way to the spin-glass behavior at low temperatures. The ferromagnetic state becomes unstable with respect to a creation of random field at low temperatures.

Figure 12 shows $M-H$ curves for $x=0.40$ at several temperatures up to $H=10 \mathrm{kOe}$. Figure 13 indicates expanded plots in low field region up to 200 Oe. A slight indication of the hysteresis with small coercive force is seen at 5.0 and $25 \mathrm{~K}$. Since the Curie-Weiss law is well applicable to the data in $T \geq T_{\mathrm{c}}$, the $\mathrm{Cr}$ atoms have a spin with a formal $\mathrm{Cr}^{3+}$ valence state, then, the saturated moment is expected to be $3.60 n_{\mathrm{B}}$ f.u. $^{-1}$ for $x=0.40$. Here the value of the magnetic moment, $n_{\mathrm{B}}$ is defined to be $n_{\mathrm{B}}=g S$ in units of $\mu_{\mathrm{B}}$, where $g$ is the Landefs $g$-factor.

The values of the magnetization at $10 \mathrm{kOe}$ for $x=0.40$ are listed in Table 3. The last column indicates the percent of $n_{\mathrm{B}} \mathrm{Cr}$-atom ${ }^{-1}$ on $n_{\mathrm{B}}=3.0$ for the saturated value of $\mathrm{Cr}^{3+}$. The value per formula unit is given as follow, the experimental values of the magnetic moment at $10 \mathrm{kOe}$ are $1.92 n_{\mathrm{B}} \mathrm{f.u}^{-1}$ at $5 \mathrm{~K}$. The value of magnetization for $x=0.40$ is given to be $1.10 \times 10^{4} \mathrm{emu}$ mol-f.u. ${ }^{-1}$ at $H=10 \mathrm{kOe}$. This magnitude leads to the magnetic moment $n_{\mathrm{B}} \mathrm{Cr}^{-a_{0}}{ }^{-1}$ in dimensionless numerical value. Here $n_{\mathrm{B}} \mathrm{Cr}^{-a t o m}{ }^{-1}=g S$ $=M /\left(1.20 \times N_{\mathrm{A}} \times \mu_{\mathrm{B}}\right)=1.60 \mathrm{Cr}^{\mathrm{atom}}{ }^{-1}$ for the case of $x=0.40$, where $M$ 


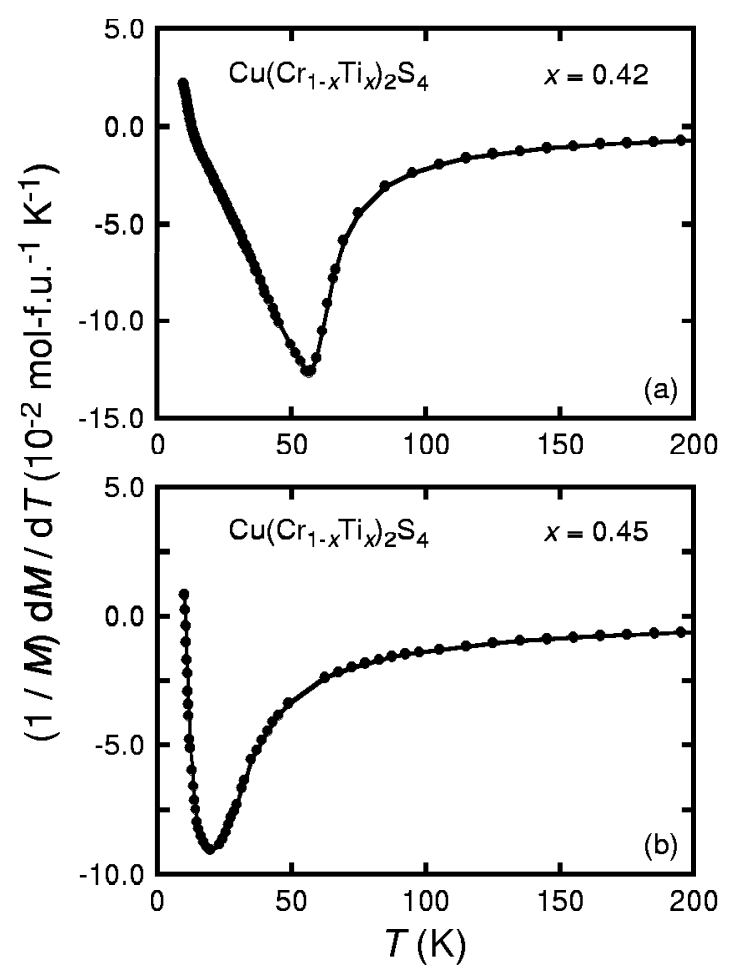

Figure 11: The temperature dependence of $(1 / M) d M / d T$ for (a) $x=0.42$ and (b) $x=$ 0.45 , which indicate negative peaks at $55 \mathrm{~K}$ and $20 \mathrm{~K}$, respectively. We adopt these values as $T_{\mathrm{c}}$ for the weakened ferromagnets. 


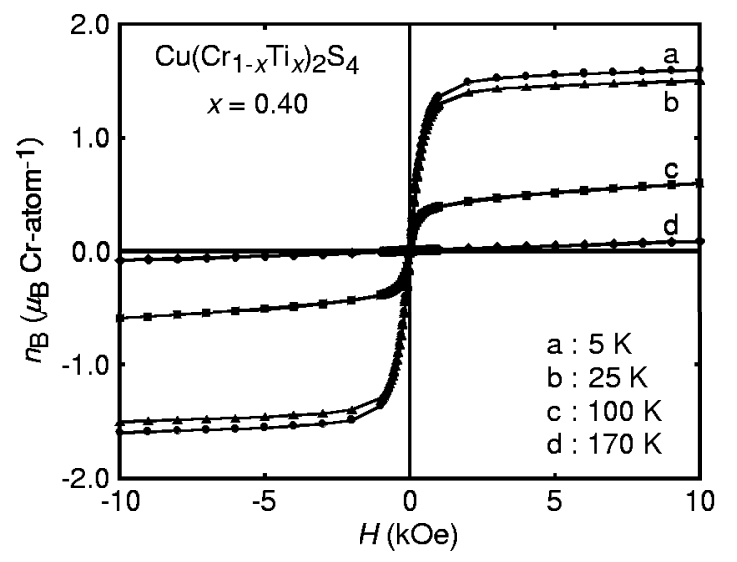

Figure 12: Magnetization as a function of applied field up to $10.00 \mathrm{kOe}$ for $x=0.40$ at several temperatures in the ZFC process. 


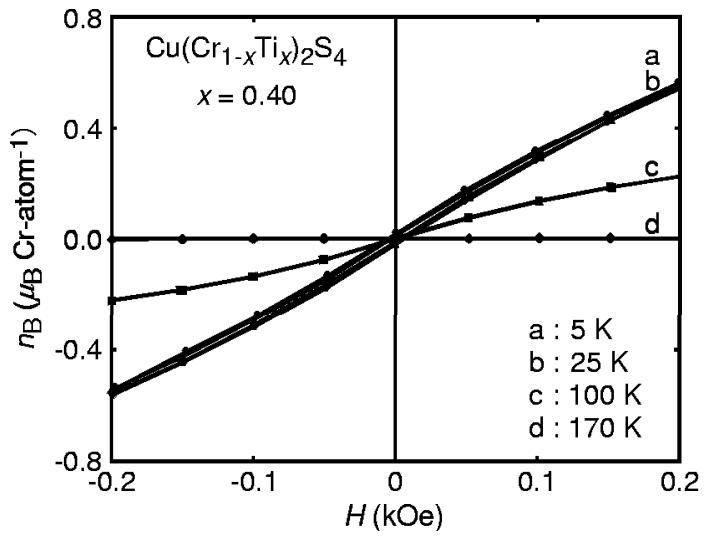

Figure 13: An enlargement of data of Fig. 12 in low field part up to 200 Oe for $x=0.40$ at several temperatures in the ZFC process. 
is the moment of f.u.-mol ${ }^{-1}$, and $N_{\mathrm{A}}$ is the Avogadro's number.

The magnitude of $n_{\mathrm{B}} \mathrm{Cr}_{\text {-atom }}{ }^{-1}$ is only $53 \%$ the value of $S=3 / 2$. The magnetic order is not collinear spin alignment and the moment is not saturated at $10 \mathrm{kOe}$ at $5.0 \mathrm{~K}$. The magnetic frustration occurs at low temperatures with the competition between ferro- and antiferromagnetic interactions. These numerical values in the re-entrant regime at low temperatures indicates that the conventional domain-wall blocking like in standard ferromagnets does not occur, but the mechanism originated from the local frustration influenced the $M-H$ curves with small values of coercive force $H_{\mathrm{c}}$.

\subsection{Spin-glass and the G-T model}

The gradual variation of the magnetization implies that $\mathrm{Ti}$ substitution for $\mathrm{Cr}$ can cause the competing interaction between ferromagnetic and antiferromagnetic interactions, as can be seen in Figs. $3-6$. The predominant ferromagnetic state appears in the range of $x \leq 0.35$. The ferromagnetic state disappears at the critical composition $x_{c}=0.47$. The re-entrant transition is characterizes by a drop of the magnetization at lower temperature for the specimens of $0.40 \leq x \leq 0.47$. Figures 4 and 5 display the spinglass characteristics with the irreversibility between the $\mathrm{ZFC}$ and the $\mathrm{FC}$ magnetizations. The interaction between nearest-neighbor (n.n.) $\mathrm{Cr}-\mathrm{S}-\mathrm{Cr}$ is ferromagnetic because of $90^{\circ}$ superexchange interaction and the interactions between next nearest neighbor (n.n.n.) would be antiferromagnetic. These ferro- and antiferromagnetic- competing interactions lead to the spin frustration. Since the n.n.n. antiferromagnetic interactions are not enough strong to compete the n.n. ferromagnetic one, it is expected that the substitution of $\mathrm{Ti}$ weakens gradually the strength of the inherent n.n. ferromagnetic interaction with increasing the value of $x$. Consequently in spin-glass composition region with higher value of $x$, the antiferromagnetic bonds are sufficient to destroy the long-range ferromagnetic order.

A striking feature emerges, that is, two characteristic temperatures exist below $T_{\mathrm{c}}$ in an intermediate composition region $x=0.42$ in Fig. 4 and $x=$ 0.45 in Fig. 5. One is the temperature at which the magnetization shows the peak, while the other is that the irreversibility arises between the $\mathrm{ZFC}$ and the FC magnetization decreasing temperature. These results are interpreted satisfactorily by the G-T theory.(44) Let $T_{\mathrm{GT}}$ be the temperature defined as that at which the magnetization reaches a maximum. Furthermore, we take and define the $T_{\mathrm{AT}}$ as the splitting temperature between the $\mathrm{ZFC}$ and 
the FC magnetization with decreasing temperature.(47) The G-T theory is performed on the basis of Heisenberg isotopic vector spin model and therefore it provides more realistic model than other theories concerned with Ising spin model.(45; 46) The G-T theory predicts the mixed phases below $T_{\mathrm{GT}}$, where ferromagnetism and spin-glass orders coexist, due to freezing of the transverse components of the spins to the spontaneous magnetization direction in the absence of external magnetic field.

In the actual experimental situation in the presence magnetic field, the transverse $x$ - and $y$-components of the spins indicate the inside plane perpendicular to the applied field. The microscopic direct evidence of the freezing of the transverse-spin components is not given because the only macroscopic magnetization measurements have been obtained. This mixed coexistence phase is labeled here as SGI phase. It is noted that the coexistence takes place everywhere in the specimen, which is nothing to do with a spatially segregated coexistence. The $T_{\mathrm{AT}}$ is the temperature below which the other new state arises with strong irreversibility between the $\mathrm{ZFC}$ and the FC magnetization, labeled here as SGII phase. Strictly speaking, de Almeida and Thouless have considered the the Ising spin model, nevertheless the SGII phase of G-T theory corresponds exactly to that of instability line of spontaneous breaking of the replica symmetry in A-T theory. $(44 ; 47)$

For $x \geq 0.47$, the temperatures $T_{\mathrm{GT}}$ coincides with $T_{\mathrm{AT}}$, hereafter labeled as $T_{\mathrm{g}}$. The typical spin-glass temperature dependence is seen for $x=0.47$ and $x=0.50$ in Fig. 5, such as the plateau for the FC magnetization as a function of temperature below $T_{\mathrm{g}}$. The values of the asymptotic Weiss temperature $\theta$ are extremely low for $x=0.47$ and 0.50 .

The spin-glass like behavior is found for the composition over $0.55 \leq$ $x \leq 0.85$ as shown in Fig. 6. The temperature dependences of $M$ is more complicated, even though the behavior looks like a diluted spin-glass. The FC magnetization is not a plateau but depends on temperature below $T_{\mathrm{g}}$. It is important to note that the splitting between the $\mathrm{ZFC}$ and the $\mathrm{FC}$ curves begins around at $T_{\mathrm{g}}$, independent of the composition.

The maximum value of magnetization does not vary monotonically over the range of $0.47 \leq x \leq 0.85$ in spin-glass regime, as seen in Figs. 5 and 6. That is, this maximum height increases from $x=0.47$ to 0.60 , then, from $x=0.60$ to 0.85 that decreases. The clear interpretation for this nonmonotonic variation cannot be given at present. Presumably, a main origin comes from the change of the valence state of Cr ions on an assumption that the formal valence state of $\mathrm{Ti}$ ions is $4+$ with non magnetic state. The 
formal valence of $\mathrm{Cr}$ ions changes at $x=0.50 \mathrm{from} \mathrm{Cr}^{3+} / \mathrm{Cr}^{4+}$ for $x \leq 0.50$ to $\mathrm{Cr}^{2+} / \mathrm{Cr}^{3+}$ for $0.50 \leq x \leq 0.75$. Furthermore, it changes at $x=0.75$ from $\mathrm{Cr}^{2+} / \mathrm{Cr}^{3+}$ to $\mathrm{Cr}^{+} / \mathrm{Cr}^{2+}$ for $0.75 \leq x \leq 0.83$. For $x \geq 0.83$, the formal valence state does not make sense any more because of the no valence. The spin values of $\mathrm{Cr}$ ions are $S=1,3 / 2,2,5 / 2$, for $\mathrm{Cr}^{4+}, \mathrm{Cr}^{3+}, \mathrm{Cr}^{2+}$, and $\mathrm{Cr}^{+}$, respectively, with different moments. Possibility of the formation of the spin clusters may become larger due to this valence changes in the diluted spin glass regime. The evaluation of the non-monotonic variation of $M$ over the range of $0.47 \leq x \leq 0.85$ is in a hard situation and runs into difficulty.

Specimens with $x \geq 0.90$ remain paramagnetic down to $2.0 \mathrm{~K}$ and display no overt trace of spin-glass. The decrease of magnetic concentration gives rise to the reduction of interaction.

\subsection{Magnetic phase diagram}

Now we are going to construct a magnetic phase diagram of $\mathrm{Cu}\left(\mathrm{Cr}_{1-x} \mathrm{Ti}_{x}\right)_{2} \mathrm{~S}_{4}$. The magnetic phase diagram between temperature $T$ versus Ti-composition $x$ has been obtained experimentally as illustrated in Fig. 14. Figure 15 displays an enlargement of the data over the composition range of $0.40 \leq x \leq 0.70$. The ferromagnetic transition temperature decreases abruptly from $T_{\mathrm{c}} \simeq 380$ $\mathrm{K}(x=0.00)$ to the less than $10 \mathrm{~K}(x=0.47)$. The pure ferromagnetic state is perturbed gradually by $\mathrm{Ti}$ substitution. The clear predominant ferromagnetic transition is seen for only $x \leq 0.35$.

For the composition range of $0.40 \leq x \leq 0.47$, one can see, with decreasing temperature, first a transition at the $T_{\mathrm{c}}$ from a paramagnetic to a ferromagnetic state, a second transition at the $T_{\mathrm{GT}}$ from the ferromagnetic to a SGI phase where the ferromagnetism and spin-glass coexist due to freezing of the transverse-spin components of Heisenberg model, then finally a third transition at the $T_{\mathrm{AT}}$ from the SGI phase to a SGII phase where the $x^{-}, y^{-}$, and $z$-components of spin freeze, indicating the strong irreversibility between the $\mathrm{ZFC}$ and the $\mathrm{FC}$ magnetizations. $(44 ; 47)$

Three characteristic temperatures of $T_{\mathrm{c}}, T_{\mathrm{GT}}$ and $T_{\mathrm{AT}}$ merge into $7.40 \mathrm{~K}$ at $x=0.47$ in $H=100$ Oe. This is a multicritical point as illustrated in Figs. 14 and 15. A basic issue is to observe the variations of the $\mathrm{G}-\mathrm{T}$ and the $\mathrm{A}-\mathrm{T}$ lines as a function of applied field and detect any field effect on these lines, however, we have not yet measured the field dependence of the $\mathrm{G}-\mathrm{T}$ and $\mathrm{A}-\mathrm{T}$ lines. As seen in Fig. 14, as decreasing the composition $x$ from $x=0.40$ to lower one, the $\mathrm{G}$ - $\mathrm{T}$ and the $\mathrm{A}$ - $\mathrm{T}$ lines becomes ill defined. The $\mathrm{G}-\mathrm{T}$ and A-T lines vanish asymptotically with a crossover behavior when the system 


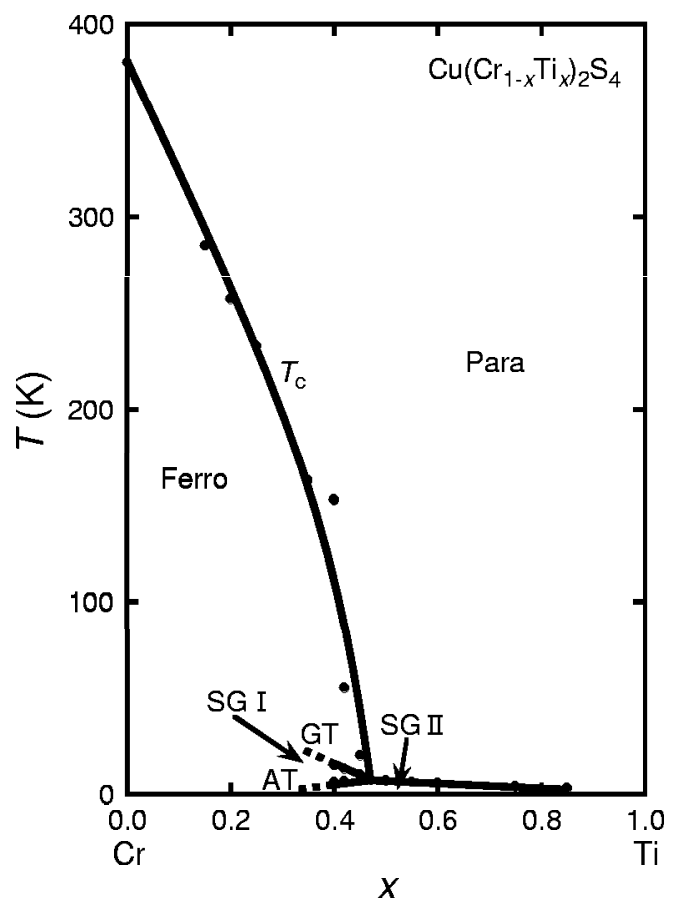

Figure 14: Magnetic phase diagram for $\mathrm{Cu}\left(\mathrm{Cr}_{1-x} \mathrm{Ti}_{x}\right)_{2} \mathrm{~S}_{4}$ at a constant field of 100 Oe. The solid curves give a guide to the eye: para, paramagnetic; ferro, ferromagnetic; SGI, spin-glass I (GT-phase); SGII, spin-glass II(AT-phase). 


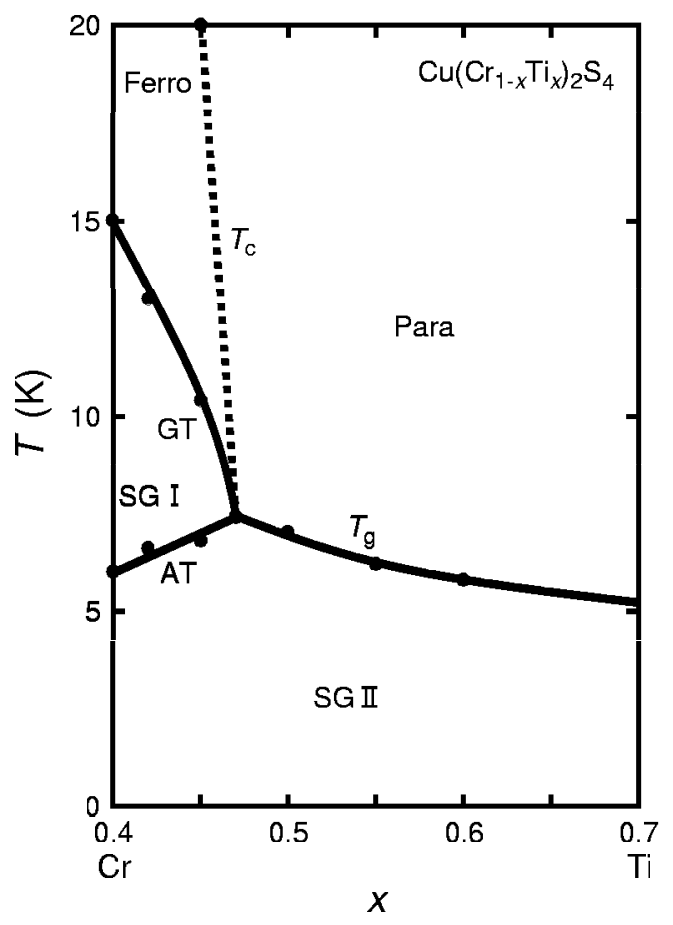

Figure 15: An enlargement of data of Fig. 14. The magnetic phase diagram for $\mathrm{Cu}\left(\mathrm{Cr}_{1-x} \mathrm{Ti}_{x}\right)_{2} \mathrm{~S}_{4}$ at a constant field of $100 \mathrm{Oe}$ is mapped over the range $0.40<x<0.70$. The lines are a visual guide. Three characteristic temperatures of $T_{\mathrm{c}}, T_{\mathrm{GT}}$, and $T_{\mathrm{AT}}$ merge into $7.40 \mathrm{~K}$ at $x=0.47$ in $H=100$ Oe. This is a multicritical point. 
approaches, from the coexistence phases between the ferromagnetic and the spin-glass phases, to the predominant ferromagnetic phase with decreasing $x$. This behavior is consistent with the prediction by the theories. $(44 ; 47)$ The region of $0.40 \leq x \leq 0.47$ has served the characteristic information on the problem of the $\mathrm{G}-\mathrm{T}$ and $\mathrm{A}-\mathrm{T}$ transitions.

The problem of the coexistence of the ferromagnetic and the spin-glass phases has been less extensively investigated so far, in particular experimental point of view. The global aspects of our experimental results has been well explained in terms of $\mathrm{G}-\mathrm{T}$ and $\mathrm{A}$-T theories. $(44 ; 47 ; 61)$ Overall features of the magnetic phase diagram is understood. Here we would like to express our speculation along with some of present results, concerned with the ac susceptibility, $\chi_{a c}$. The out-of-phase susceptibility (imaginary part) referred to an absorption, $\chi$ ", may rise at around $T_{c}$, with decreasing temperature. Further $\chi$ " exhibits two characteristic temperatures, corresponding to the $T_{\mathrm{GT}}$ and the $T_{\mathrm{AT}}$ in the restricted composition range over $0.40 \leq x \leq 0.47$. These behaviors will indicate manifestly to the freezing of transverse $x$ - and $y$-components of the isotropic Heisenberg spin at G-T line, and finally to the $z$-component at A-T line.

The typical spin-glass behavior is observed such as the existence of plateau for FC magnetization as a function of temperature below $T_{\mathrm{g}}$ for $x=0.47$ and 0.50 . The high temperature asymptotic Weiss temperature $\theta$ is the extremely low value, as seen in Fig. 7 and Table 1.

Over a wide composition range of $0.55 \leq x \leq 0.85$, the spin-glass like behavior is observed, which is called here a diluted spin-glass. The FC curves below $T_{\mathrm{g}}$ was not a plateau. In higher composition $x \geq 0.50$, the magnetic concentration of $\mathrm{Cr}$ moment is lowered, therefore, isolated clusters of spin remain. Inside the clusters, the spin correlated strongly. For different $x$, the cluster sizes and the evolution of short-range order become complicated on changing temperature. As the temperature is lowered, larger clusters are firstly blocked, then smaller clusters freeze, which are large enough to be frozen at that temperature. These coalesce of clusters causes the departure from the typical spin-glass behavior in the temperature dependence of $M$. Presumably the behavior below $T_{\mathrm{g}}$ without the plateau of the $\mathrm{FC}$ magnetization might attributed to the influence of the spin clusters. The strange composition dependence of the Weiss temperature $\theta$ as seen in Fig. 9 and the deviation from the Curie-Weiss law comes from the same origin of the spin clusters, as illustrated in Figs. 8 and 9 . For $x \geq 0.90$, the cusp as spin-glass is never found and the difference between the $\mathrm{ZFC}$ and the $\mathrm{FC}$ is not seen 
down to $2.0 \mathrm{~K}$.

\section{Summary}

(1) It is generally believed that a simple diluted ferromagnetism appears as increasing the nonmagnetic composition. The system of $\mathrm{Cu}\left(\mathrm{Cr}_{1-x} \mathrm{Ti}_{x}\right)_{2} \mathrm{~S}_{4}$, on the contrary, leads to a completely different behavior. It reflects the richness and subtleties of the magnetic interaction even though the nonmagnetic Ti substitution for $\mathrm{Cr}$ atoms is executed. (2) A magnetic phase diagram of the system $\mathrm{Cu}\left(\mathrm{Cr}_{1-x} \mathrm{Ti}_{x}\right)_{2} \mathrm{~S}_{4}$ between temperature versus $\mathrm{Ti}$ composition $x$ has been established experimentally. This diagram is in general agreement with the theoretical prediction by Gabay and Toulouse.(44) The interpretation of the results is primitive, without consistent microscopic picture. (3) It is turned out that the $\mathrm{Ti}$ substitution can cause the magnetic frustration originated from the competition between ferromagnetic and antiferromagnetic interactions. (4) The G-T line has been identified experimentally, at which the maximum magnetization is observed in the intermediate composition region. This G-T line corresponds to the freezing of the transverse degree of freedom based on the Heisenberg isotropic vector spin model below which the re-entrant mixed phases exist. (5) The A-T line is referred to the temperature of the splitting due to an irreversibility between the ZFC and the FC magnetizations. (6) The multi critical point is proved to be $x=$ 0.47 and $T=7.40 \mathrm{~K}$ in 100 Oe, at which the ferromagnetic, the spin-glass, and the paramagnetic states become unstable. (7) Systematic measurements of thermal and electrical properties are expected. An ac susceptibility $\chi_{a c}$ measurement is an important tool to study the dynamical properties of the disordered system. We are currently carrying out these studies and will publish elsewhere. A microscopic elucidation is also required.

The authors would like to thank Mr. S. Maruichi for his valuable experimental collaboration.

\section{References}

[1] H. Hahn, C. de Lorent, and B. Harder, Z. Anorg. Allg. Chem. 283 (1956) 138. 
[2] F. K. Lotgering, in Proc. the Int. Conf. on Magnetism, Nottingham (Institute of Physics and Physical Society, London, 1964), p. 533.

[3] F. K. Lotgering, Solid State Commun. 2 (1964) 55.

[4] R. J. Bouchard, P. A. Russo, and A. Wold, Inorg. Chem. 4 (1965) 685.

[5] P. M. Raccah, R. J. Bouchard, and A. Wold, J. Appl. Phys. 37 (1966) 1436.

[6] M. Robbins, H. W. Lehmann, and J. G. White, J. Phys. Chem. Solids 28 (1967) 897.

[7] K. Ohbayashi, Y. Tominaga, and S. Iida, J. Phys. Soc. Jpn. 24 (1968) 1173.

[8] J. B. Goodenough, J. Phys. Chem. Solids 30 (1969) 261.

[9] M. Robbins, A. Menth, A. Miksovsky, and R.C. Sherwood, J. Phys. Chem. Solids. 31 (1970) 423.

[10] R. P. van Stapele and F. K. Lotgering, J. Phys. Chem. Solids 31 (1970) 1547 .

[11] T. Kanomata, H. Ido, and T. Kaneko, J. Phys. Soc. Jpn. 29 (1970) 332.

[12] M. Robbins, A. Menth, M. A. Miksovsky, and R. C. Sherwood, J. Phys. Chem. Solids 31 (1970) 423.

[13] Y. Wada and K. Ametani, Therm. Acta 2 (1971) 237.

[14] E. Riedel and E. Horváth, Z. Anorg. Allg. Chem. 399 (1973) 219.

[15] K. P. Belov, Yu. D. Tretfyakov, I. V. Gordeev, L. I. Koroleva, A. V. Pedfko, E. I. Smirnovskaya, V. A. Alferov, and Yu. G. Saksonov, Fiz. Tverd. Tela (Leningrad) 14 (1972) 2155 [Sov. Phys. Solid State 14 (1973) 1862 .

[16] Yu.D. Tretyakov, I.V. Gordeev, and Ya.A. Kesler, J. Solid State Chem. 20 (1977) 345.

[17] H. Itoh, J. Phys. Soc. Jpn. 48 (1980) 1130. 
[18] J. Padiou, D. Bideau, and J. P. Troadec, J. Solid State Chem. 31 (1980) 401.

[19] Ferro-Magnetic Materials, edited by E. P. Wohlfarth, A handbook on the properties of magnetically ordered substances Vol.3 (North-Holland, Amsterdam, 1982), p603.

[20] J. Horikawa, T. Hamajima, F. Ogata, T. Kambara, and K. Gondaira, J. Phys. C: Solid State Phys. 15 (1982) 2613.

[21] H. D. Lutz, U. Koch, and I. Okonska-kozlowska, J. Solid State Chem. 51 (1984) 69.

[22] J. Krok-Kowalski, J. Warczewski, and K. Nikiforov, J. Alloys Compd. 315 (2001) 62.

[23] A. Kimura, J. Matsuno, J. Okabayashi, A. Fujimori, T. Shishidou, E. Kulatov, and T. Kanomata, Phys. Rev. B 63 (2001) 224420.

[24] K. Oda, S. Yoshii, Y. Yasui, M. Ito, T. Ido, Y. Ohno, Y. Kobayashi, and M. Sato, J. Phys. Soc. Jpn. 70 (2001) 2999.

[25] M. Muroi, R. Street, and P.G. McCormick, Phys. Rev. B 63 (2001) 0.52412 .

[26] R. Endoh, J. Awaka, and S. Nagata, Phys. Rev. B 68 (2003) 115106.

[27] K.-H. Hellwege, A.M. Hellwege (Eds.), Landort-Börnstein, New Series, Group II, Vol. 8, Springer, Berlin, 1976, p. 27.

[28] F.J. DiSalvo and J.V. Waszczak, Phys. Rev. B 26 (1982) 2501.

[29] D. Mahl, J.Pickardt, and B. Reuter, Z. anorg. Allg. Chem. 491 (1982) 203.

[30] T. Koyama, H. Sugita, S. Wada, K. Miyatani, T. Tanaka, and M. Ishikawa, Physica B 284-288 (2000) 1513.

[31] N. Matsumoto, T. Hagino, K. Taniguchi, S. Chikazawa, S. Nagata, Physica B 284-288 (2000) 1978.

[32] H. Okada, K. Koyama, and K. Watanabe, J. Alloys Compd. 403 (2005) 34. 
[33] F.K. Lotgering and R.P. van Stapele, Solid State Commun. 5 (1967) 143.

[34] J. B. Goodenough, Solid State Commun. 5 (1967) 577.

[35] F.K. Lotgering and R.P. van Stapele, J. Appl. Phys. 39 (1968) 417.

[36] J. Smit, Solid State Commun. 6 (1968) 745.

[37] G. Strick, G. Eulenberger, and H. Hahn, Z. anorg. Allg. Chem. 357 (1968) 338.

[38] E. Riedel and E. Horvath, Mater. Res. Bull. 8 (1973) 973.

[39] Ya.A. Kesler, E.G. Zhukov, D.S. Filimonov, E.S. Polulyak, T.K. Menshchikova, and V.A. Fedorov, Inorg. Mater. 41 (2005) 914.

[40] F. Kariya (private communication).

[41] Y. Iijima, Y. Kamei, N. Kobayashi, J. Awaka, T. Iwasa, S. Ebisu, S. Chikazawa, and S. Nagata, Philos. Mag. 83 (2003) 2521.

[42] T. Furubayashi, H. Suzuki, N. Kobayashi, and S. Nagata, Solid State Commun. 131 (2004) 505.

[43] M. Ito, H. Yamamoto, S. Nagata, and T. Suzuki, Phys. Rev. B 74 (2006) 214412.

[44] M. Gabay and G. Toulouse, Phys. Rev. Lett. 47 (1981) 201.

[45] D. Sherrington and S. Kirkpatrick, Phys. Rev. Lett. 35 (1975) 1792.

[46] S. Kirkpatrick and D. Sherrington, Phys. Rev. B 17 (1978) 4384.

[47] J. R. L. de Almeida and D. J. Thouless, J. Phys. A 11 (1978) 983.

[48] S. Nagata, P. H. Keesom, and H. R. Harrison, Phys. Rev. B 19 (1979) 1633.

[49] K. Binder and A. P. Young, Rev. Mod. Phys. 58 (1986) 801.

[50] Cooperative Dynamics in Complex Physical Systems, edited by H. Takayama (Springer Series in Synergetics, 43, 1989). 
[51] Spin Glasses, by K. H. Fischer and J. A. Hertz (Cambridge University Press, 1991).

[52] Spin Glasses: An Experimental Introduction, by J. A. Mydosh, (Taylor and Francis, 1993).

[53] H. Kawamura, Phys. Rev. Lett. 68 (1992) 3785.

[54] B. R. Coles, B. V. B. Sarkissian, and R. H. Taylor, Phil. Mag. B 37 (1978) 489.

[55] H. Maletta and P. Convert, Phys. Rev. Lett. 42 (1979) 108.

[56] A. Berton, J. Chaussy, J. Odin, R. Rammal, J. Souletie, J. L. Tholence, and R. Tournier, J. Appl. Phys. 52 (1981) 1763.

[57] K. Katsumata, J. Magn. Magn. Mater. 31-34 (1983) 1435.

[58] H. Yoshizawa, S. Mitsuda, H. Aruga, and A. Ito, J. Phys. Soc. Jpn. 58 (1989) 1416.

[59] I. A. Campbell, S. Senoussi, F. Varret, J. Teillet, and A. Hamzić, Phys. Rev. Lett. 50 (1983) 1615.

[60] S. Iikubo, Y. Yasui, K. Oda, Y. Ohno, Y. Kobayashi, M. Sato, and K. Kakurai, J. Phys. Soc. Jpn. 71 (2002) 2792.

[61] D. J. Thouless, J. R. L. de Almeida, and J. M. Kosterlitz J. Phys. C 13 (1980) 3721, and references therein. 
Table 1 Summary of the magnetic properties of $\mathrm{Cu}\left(\mathrm{Cr}_{1-x} \mathrm{Ti}_{x}\right)_{2} \mathrm{~S}_{4}$. These numerical values are extracted from the modified Curie-Weiss law. The value of $\chi_{0}$ includes appreciable experimental errors. The effective number of magnetic moment $\mathrm{Cr}$-atom ${ }^{-1}$ indicates that only $\mathrm{Cr}$ atoms posses a localized moment.

\begin{tabular}{ccccccc}
\hline & $C$ & $\theta$ & $p_{\text {eff }}$ & $p_{\text {eff }}$ & $\chi_{0}$ & Temperature \\
\cline { 2 - 7 }$x$ & $\begin{array}{c}(\text { emu K } \\
\left.\text { mol-f.u. }^{-1}\right)\end{array}$ & $(\mathrm{K})$ & $\left(\right.$ f.u. $\left.^{-1}\right)$ & $\left(\right.$ Cr-atom $\left.^{-1}\right)$ & $($ emu mol & \\
& 2.09 & 273 & 4.09 & 3.23 & $-1.39 \times 10^{-3}$ & $315-350$ \\
\hline 0.20 & 2.05 & 166 & 4.05 & 3.55 & $-4.94 \times 10^{-4}$ & $200-350$ \\
0.35 & 1.58 & 144 & 3.56 & 3.25 & $+6.22 \times 10^{-4}$ & $190-300$ \\
0.40 & 2.03 & 55.8 & 4.03 & 3.74 & $-4.00 \times 10^{-4}$ & $140-300$ \\
0.42 & 2.00 & 27.0 & 4.00 & 3.82 & $-9.19 \times 10^{-4}$ & $90-300$ \\
0.45 & 1.97 & 0.57 & 3.97 & 3.86 & $-5.49 \times 10^{-4}$ & $160-300$ \\
0.47 & 2.13 & 1.12 & 4.13 & 4.13 & $-9.73 \times 10^{-4}$ & $50-300$ \\
0.50 & 2.00 & 4.38 & 4.00 & 4.22 & $-1.35 \times 10^{-3}$ & $20-300$ \\
0.55 & 1.55 & 26.7 & 3.52 & 3.93 & $-3.83 \times 10^{-4}$ & $100-300$ \\
0.60 & 0.95 & 37.6 & 2.75 & 3.89 & $-2.71 \times 10^{-4}$ & $120-300$ \\
0.75 & 0.57 & 29.8 & 2.13 & 3.90 & $-1.92 \times 10^{-4}$ & $100-300$ \\
0.85 & 0.38 & 20.4 & 1.75 & 3.92 & $-4.49 \times 10^{-5}$ & $120-300$ \\
0.90 & 0.21 & 12.4 & 1.30 & 4.11 & $-1.59 \times 10^{-5}$ & $20-300$ \\
0.95 & & & & & & \\
\hline
\end{tabular}


Table 2 The temperature indicating the magnetic phase boundary. The value of $(1 / M)(\mathrm{d} M / \mathrm{d} T)$ shows the temperature derivative which is normalized by the magnitude of $M$. We adopt the sharp negative peak tempperature of $(1 / M)(\mathrm{d} M / \mathrm{d} T)$ as $T_{\mathrm{c}}$, see text.

\begin{tabular}{|c|c|c|c|c|}
\hline$x$ & $\begin{array}{c}\text { Temperature of the } \\
\text { negative peak for } \\
\mathrm{d} M / \mathrm{d} T \\
\text { (mere calculations) }\end{array}$ & $\begin{array}{c}T_{\mathrm{c}} \\
\text { Temperature of the } \\
\text { negative peak for } \\
(1 / M)(\mathrm{d} M / \mathrm{d} T)\end{array}$ & $T_{\mathrm{GT}}$ & $\begin{array}{c}T_{\mathrm{AT}} \\
\left(T_{\mathrm{g}} \text { for } x \gtrsim 0.47\right)\end{array}$ \\
\hline & $(\mathrm{K})$ & $(\mathrm{K})$ & $(\mathrm{K})$ & $(\mathrm{K})$ \\
\hline 0.15 & 279 & 285 & & \\
\hline 0.20 & 249 & 257 & & \\
\hline 0.25 & 218 & 233 & & \\
\hline 0.35 & 129 & 163 & & \\
\hline 0.40 & 93 & 153 & 15.0 & 6.0 \\
\hline 0.42 & 31 & 55 & 13.0 & 6.6 \\
\hline 0.45 & 15 & 20 & 10.4 & 6.8 \\
\hline 0.47 & 9.3 & 9.3 & 7.4 & 7.4 \\
\hline 0.50 & & & & 7.0 \\
\hline 0.55 & & & & 6.2 \\
\hline 0.60 & & & & 5.8 \\
\hline 0.75 & & & & 4.0 \\
\hline 0.85 & & & & 3.2 \\
\hline 0.90 & & & & \\
\hline 0.95 & & & & \\
\hline
\end{tabular}


Table 3 The numerical value of magnetic moment $n_{\mathrm{B}}=g S=2 S$ for $x=0.40$ of $\mathrm{Cu}\left(\mathrm{Cr}_{1-x} \mathrm{Ti}_{x}\right)_{2} \mathrm{~S}_{4}$, where $g$ is the Lande's $g$-factor. These results are obtained at $H=10.00 \mathrm{kOe}$ at several temperatures. The last column indicates the percent of $n_{\mathrm{B}} \mathrm{Cr}$-atom ${ }^{-1}$ on $n_{\mathrm{B}}=3.00$ for the saturated value of $\mathrm{Cr}^{3+}$.

\begin{tabular}{cccc}
\hline$T$ & $n_{\mathrm{B}}$ & $n_{\mathrm{B}}$ & percent \\
\hline$(\mathrm{K})$ & $\left(\mu_{\mathrm{B}}\right.$ f.u. $\left.^{-1}\right)$ & $\left(\mu_{\mathrm{B}}\right.$ Cr-atom $\left.^{-1}\right)$ & $\%$ \\
\hline 5 & 1.92 & 1.60 & 53.2 \\
25 & 1.80 & 1.50 & 50.1 \\
100 & 0.72 & 0.60 & 19.9 \\
170 & 0.10 & 0.09 & 2.84 \\
\hline
\end{tabular}

